\title{
Lymphoma Response to Immunomodulatory Therapy Criteria Indeterminate Response 3
}

National Cancer Institute

\section{Source}

National Cancer Institute. Lymphoma Response to Immunomodulatory Therapy Criteria Indeterminate Response 3. NCI Thesaurus. Code C153155.

An increase in F-fluorodeoxyg lucose (FDG) uptake of one or more lesions without a concomitant increase in lesion size or number. 